\title{
Caso radiológico para diagnóstico
}

\section{Drs. Ignacio Martín G, Rosario Vidal T, Rodrigo Blanco H, Aurora Martín S.}

Servicio de Radiodiagnóstico. Hospital Virgen de la Concha. Zamora. España.

Servicio de Oncología Médica. Hospital Universitario de Salamanca. Salamanca. España.

\section{Historia clínica}

Mujer de 42 años, que acude a consulta de cirugía, remitida por el servicio de ginecología, por presentar masa intraabdominal en hipogastrio y fosa ilíaca derecha de unos tres años de evolución.

La exploración física puso de manifiesto un abdomen blando y depresible, no doloroso, sin palpar masas ni visceromegalias. Los exámenes de laboratorio no revelan alteraciones significativas.

Inicialmente se realizó ecografía abdominal (Figura 1), posteriormente tomografía computada (TC) (Figura 2) y resonancia magnética $(\mathrm{RM})$ (Figura 3 ).

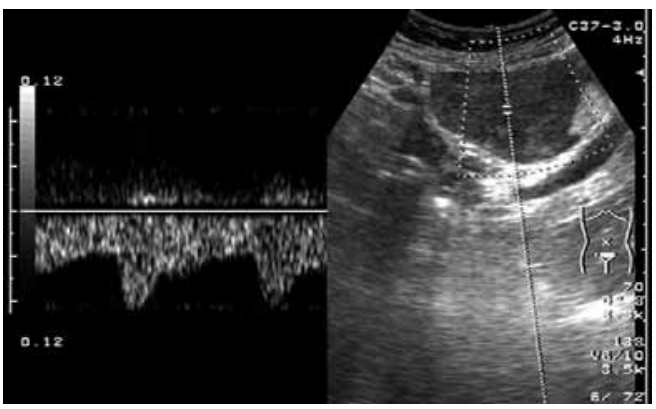

Figura 1.

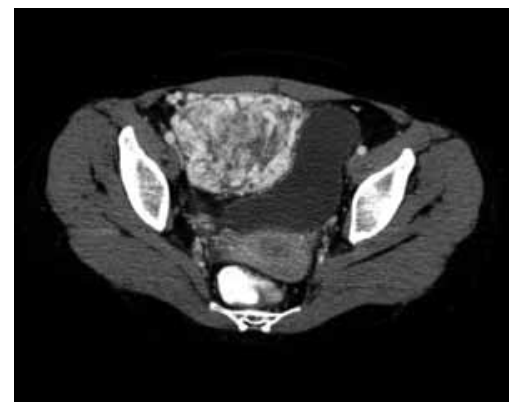

Figura 2a.

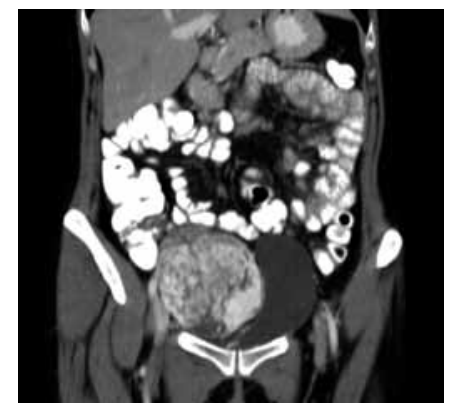

Figura $2 b$.

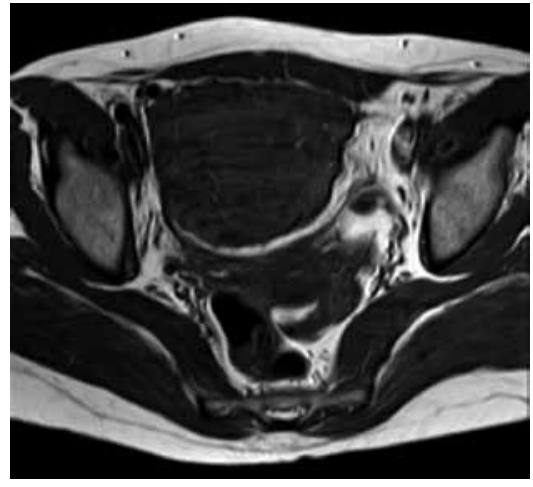

Figura 3a.

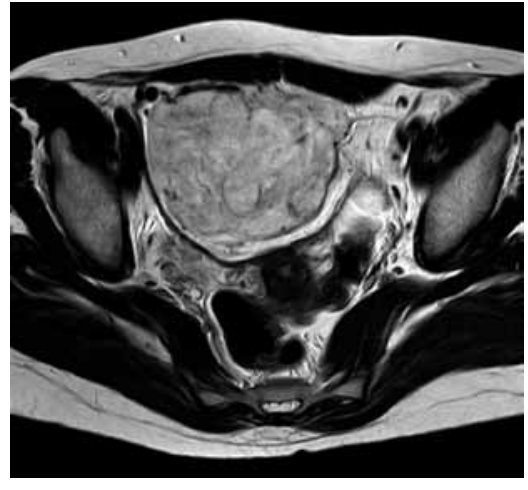

Figura 3b.

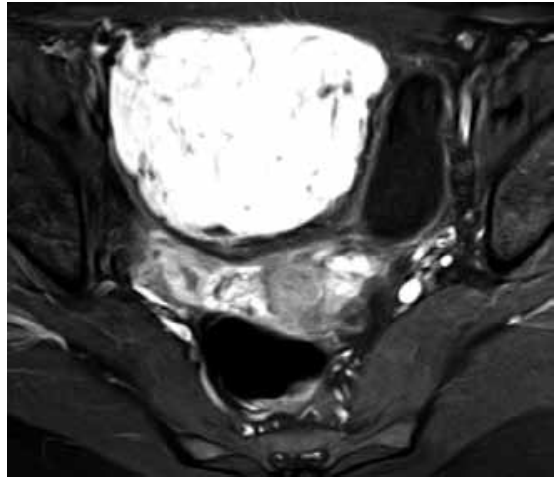

Figura 3c. 


\section{Resultado caso radiológico para diagnóstico}

\section{Drs. Ignacio Martín G, Rosario Vidal T, Rodrigo Blanco H, Aurora Martín S.}

Servicio de Radiodiagnóstico. Hospital Virgen de la Concha. Zamora. España.

Servicio de Oncología Médica. Hospital Universitario de Salamanca. Salamanca. España.

viene de la página 153.

\section{Diagnóstico:}

Hemangiopericitoma pélvico

La ecografía (Figura 1) demostró una masa de ecogenicidad heterogénea con flujo arterial en su interior, localizada en hipogastrio. La TC (Figura 2) puso de manifiesto la existencia de una masa sólida hipervascular, con áreas de necrosis, de unos $7,5 \mathrm{x}$ $9,5 \times 10,5 \mathrm{~cm}$, que parece depender de vasos ilíacos con bordes bien definidos que desplaza lateralmente la vejiga, cranealmente las asas ileales y con hipertrofia de arteria epigástrica derecha.

Posteriormente se realizó una RM, que demostró la existencia de la masa, con las relaciones anatómicas descritas en la TC, mostrando una intensidad de señal similar al músculo y homogéneo en T1. En T2 existe un aumento de señal con áreas de vacío de señal por artefactos de flujo y un intenso realce homogéneo tras la inyección de gadolinio.

Dados estos hallazgos se decide resolución quirúrgica, que consistió en resección total de la masa suprapúbica.

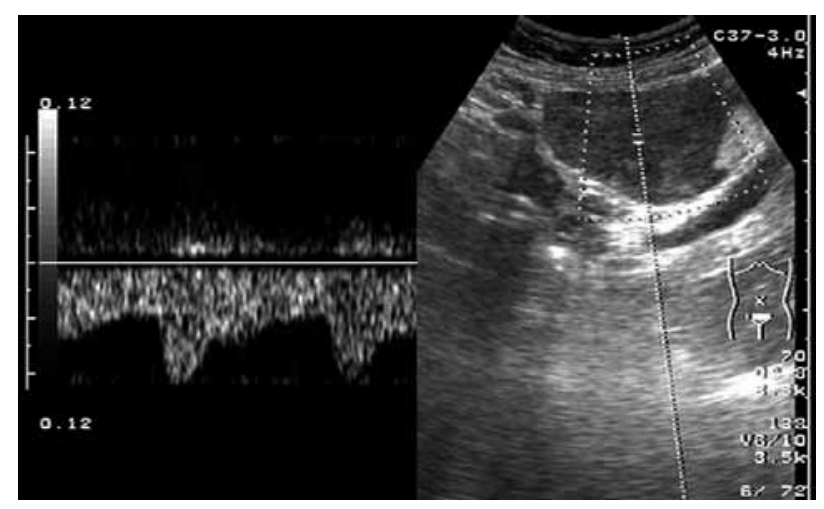

Figura 1. Ecotomografía pelviana: Masa de ecoestructura heterogénea, en hipogastrio, con flujo arterial en su interior.
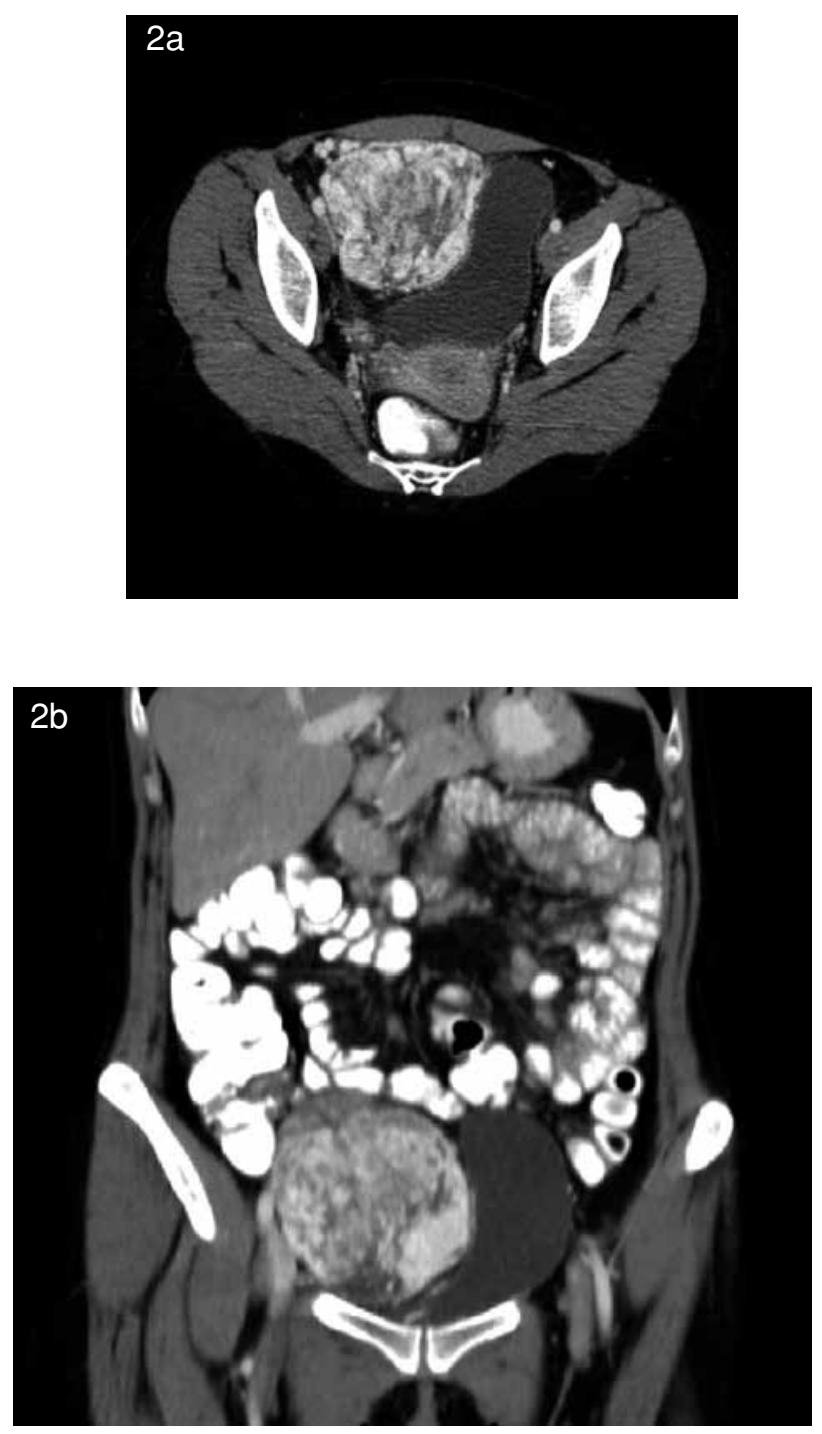

Figura 2. Tomografía computada a) Masa sólida hipervascular en fosa ilíaca derecha, con áreas de necrosis, que parece depender de vasos ilíacos con bordes bien definidos que desplaza lateralmente la vejiga y cranealmente las asas ileales. b) Reformateo coronal donde se visualizan mejor los hallazgos descritos. 

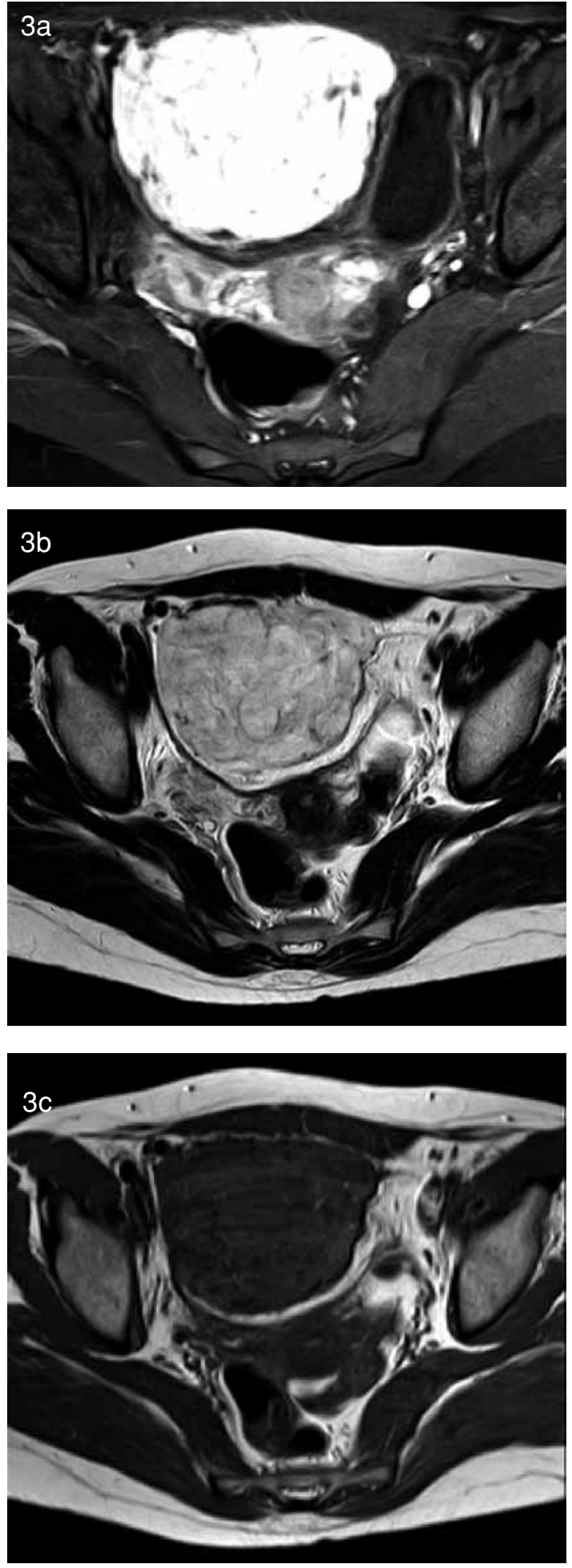

Figura 3. a) RM axial T1 donde se aprecia la masa homogénea e isointensa con el músculo. b) RM axial T2 donde existe un aumento de señal con áreas de vacío de señal por artefactos de flujo. c) RM axial tras administración de gadolinio: importante realce de la lesión, por captación de contraste.

La anatomía patológica (Figura 4) informó una lesión irregular ovoide, de superficie lisa, bien circunscrita, de coloración violácea con marcada vascularización y que en los cortes seriados presenta superficie de color marrón con trabéculas fibrosas. Las tinciones inmunohistoquímicas con vimentina y CD 34 fueron positivas, siendo negativas para $C D$ 31, S-100, desmina, actina y CD 99.

El diagnóstico fue de hemangiopericitoma pélvico.

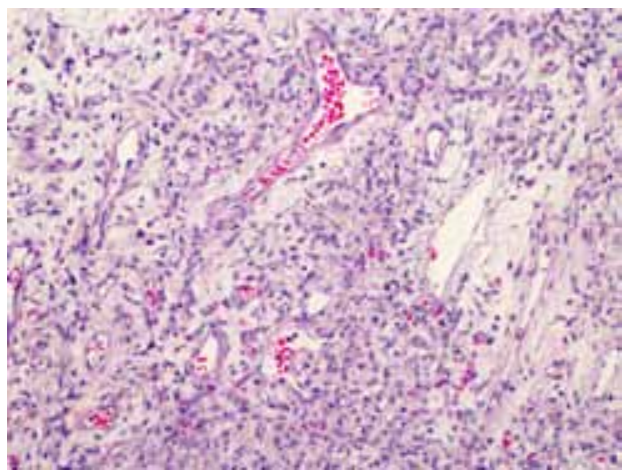

Figura 4. Anatomía patológica: Las tinciones inmunohistoquímicas con vimentina y $C D 34$ fueron positivas, siendo negativas para $C D 31, S-100$, desmina, actina y CD 99.

\section{Discusión}

El hemangiopericitoma es un raro tumor vascular que se origina en los pericitos, identificados por Zimmerman inicialmente. Los pericitos pueden dar origen a dos tumores, hemangiopericitomas y tumor de glomus.

Se localizan principalmente en la extremidad inferior, especialmente en el muslo, pelvis y retroperitoneo, son menos frecuentes en el tronco, extremidades superiores, órbitas, cabeza y cuello, intracraneales y en vísceras, sobre todo en útero y bazo(1).

Pueden aparecer a cualquier edad, siendo la edad media de aparición los 45 años, con igual distribución en ambos sexos.

Clínicamente se presentan con dolor o masa, son de lento crecimiento, provocando síntomas y signos derivados de la compresión de los órganos, como disuria, polaquiuria, nicturia, hematuria, incontinencia, hidronefrosis o constipación. Existe la posibilidad, aunque de rara aparición, de la existencia de síntomas endocrinos paraneoplásicos asociados como ginecomastia, crisis hipoglicémicas, hipertensión y osteomalacia que suelen desaparecer al extirpar el tumor(2).

Los hallazgos radiológicos del hemangiopericitoma comprenden la visualización de una masa bien definida, de gran tamaño, lobulada, con áreas de baja atenuación compatibles con necrosis y un gran realce tras la administración de contraste, como en nuestro caso. A veces se pueden apreciar calcificaciones, que serán sugerentes de malignidad. Otros signos de malignidad son las extensas áreas de necrosis y de hemorragia ${ }^{(3)}$.

El hemangiopericitoma debe considerarse en el diagnóstico diferencial de las masas de tejidos 
blandos pélvicos, si bien su frecuencia es menor que otros tumores de origen mesenquimatoso como: liposarcoma, histiocitoma fibroso maligno o leiomiosarcoma.

El rasgo más característico del hemangiopericitoma es la hipervascularización, siendo los liposarcomas preferentemente hipovasculares y con presencia de grasa. El histiocitoma fibroso maligno o leiomiosarcoma con frecuencia suelen ser hipovasculares. La histología de las diferentes tumoraciones determinan los diferentes hallazgos radiológicos, el único tumor que suele mostrar hiperintensidad en las imágenes potenciadas en $\mathrm{T} 1$ es el liposarcoma, debido a su contenido graso. Inicialmente es importante determinar si la masa es benigna o maligna para ello tendremos en cuenta el tamaño, la existencia de síntomas, en nuestro caso era una masa de larga evolución (3 años) sin síntomas, también hay que evaluar la existencia de márgenes irregulares y calcificaciones ${ }^{(2,4,5)}$.

El tratamiento del hemangiopericitoma debe ser agresivo, con extirpación total del mismo, siendo frecuente la embolización preoperatoria dado su alto riesgo de hemorragia. La radioterapia y la quimioterapia no han mostrado utilidad en el tratamiento primario, si bien se pueden emplear para los casos no resecables o metastásico y en el caso de la radioterapia ha mostrado utilidad como tratamiento adyuvante a la cirugía para mejorar el control local y las recurrencias ${ }^{(1,2)}$.
El pronóstico de estos tumores es bueno generalmente, con alta supervivencia a los 5 y 10 años (80 y $70 \%$ respectivamente) aunque requiere un seguimiento a largo plazo por la posibilidad de recurrencias o aparición de metástasis ${ }^{(2)}$.

En resumen, el hemangiopericitoma, aunque muy poco frecuente, es una lesión a tener en cuenta en el diagnóstico de las masas pélvicas, siendo sus características radiológicas más importantes la de masa bien definida, hipervascular y generalmente asintomática. Para su diagnóstico es necesario el estudio histológico.

\section{Bibliografía}

1. Pandey M, Kothri KC, Patel DD. Haemangiopericytoma: current status, diagnosis and management. Int J Urol 1997; 23: 282-285.

2. Lema J, Cimadevilla A, Rodríguez H, Durana C, Blanco M. Hemangiopericitoma retroperitoneal gigante. Actas Urol Esp. 2001; 25(9): 672-675.

3. Alpern M, Thorsen MK, Kellman GM, Pojunas K, Lawson TL. CT appearance of hemangiopericytoma. J Comput Assist Tomogr 1986; 10(2): 264-267.

4. Goldman SM, Davidson AJ, Neal J. Retroperitoneal and pelvic hemangiopericytomas: clinical, radiologic and pathologic correlation. Radiology 1988; 168(1): 13-137.

5. Nakashima J, Ueno M, Nakamura K, Tachibana M, Baba S, Deguchi N, et al. Differential diagnosis of primary benign and malignant retroperitoneal tumors. Int J Urol 1997; 4(5): 441-446.

Martin I y cols. Hemangiopericitoma pélvico. Rev Chil Radiol 2011; 17(4): 153, 195-197.

Correspondencia: Dr. Ignacio Martin G. / nachetemaga@ hotmail.com

Trabajo recibido el 10 de mayo de 2011, aceptado para publicación el 30 de mayo de 2011. 